\title{
REVIEW
}

\section{Clinical review: Volume of fluid resuscitation and the incidence of acute kidney injury - a systematic review}

\author{
John R Prowle', Horng-Ruey Chua², Sean M Bagshaw ${ }^{3}$ and Rinaldo Bellomo*4
}

\begin{abstract}
Intravenous fluids are widely administered to maintain renal perfusion and prevent acute kidney injury (AKI). However, fluid overload is of concern during AKI. Using the Pubmed database (up to October 2011) we identified all randomised controlled studies of goaldirected therapy (GDT)-based fluid resuscitation (FR) reporting renal outcomes and documenting fluid given during perioperative care. In 24 perioperative studies, GDT was associated with decreased risk of postoperative AKI (odds ratio $(O R)=0.59,95 \%$ confidence interval $(\mathrm{Cl})=0.39$ to 0.89 ) but additional fluid given was limited (median: $555 \mathrm{ml}$ ). Moreover, the decrease in AKI was greatest $(O R=0.47,95 \%$ $\mathrm{Cl}=0.29$ to 0.76$)$ in the 10 studies where FR was the same between GDT and control groups. Inotropic drug use in GDT patients was associated with decreased AKI $(\mathrm{OR}=0.52,95 \% \mathrm{Cl}=0.34$ to $0.80, P=0.003)$, whereas studies not involving inotropic drugs found no effect $(\mathrm{OR}=0.75,95 \% \mathrm{Cl}=0.37$ to $1.53, P=0.43)$. The greatest protection from AKI occurred in patients with no difference in total fluid delivery and use of inotropes $(\mathrm{OR}=0.46,95 \% \mathrm{Cl}=0.27$ to $0.76, P=0.0036)$. GDT-based FR may decrease AKI in surgical patients; however, this effect requires little overall FR and appears most effective when supported by inotropic drugs.
\end{abstract}

\section{Introduction}

Acute kidney injury (AKI) is a common condition and, even when mild, is associated with mortality and morbidity [1]. During critical illness, AKI is most frequently

*Correspondence: rinaldo.bellomo@austin.org.au

${ }^{4}$ Department of Intensive Care, Austin Health, 145 Studley Road, Heidelberg,

Victoria 3084, Australia

Full list of author information is available at the end of the article observed in the settings of sepsis and after surgery [2]. In these contexts, fluid resuscitation (FR) is almost universally employed to maintain or increase cardiac output and organ perfusion. Creatinine-related variables and urine output are often targeted during resuscitation as surrogates of adequacy of organ perfusion in general and to prevent AKI. Understanding the relationship between FR, resuscitation goals and AKI is therefore important to develop a rational approach to FR strategies in acute illness.

From a renal standpoint, fluid therapy is aimed at restoring systemic blood pressure (a major determinant of renal perfusion pressure) and cardiac output (a prerequisite for adequate renal blood flow (RBF)). Fluid administration aimed at restoring systemic blood pressure works mechanistically by increasing the preload and stroke volume. In hypovolaemia, fluid therapy restores right ventricular end-diastolic volume and is an essential first step in resuscitation. Unfortunately, conventional goals of FR and restoration of blood pressure, central venous pressure and/or urine output are only indirect measures of cardiac output and are much less indicative of the restoration of adequate organ blood flow. Similarly, the effects of critical illness, pre-existing chronic disease and pharmacotherapy can unpredictably alter physiological determinants of fluid responsiveness. These effects make the effects of FR variable in extent and duration, and make the assessment of adequacy of FR very challenging.

Evidence regarding FR and outcomes in critical illness and after major surgery can be divided into two categories. Firstly, a considerable body of evidence suggests that positive fluid balances are associated with increased postoperative complications [3,4], while more conservative intravenous fluid administration strategies do not seem harmful to renal function [5]. Similarly in critical illness, more positive fluid balances have been associated with the development of AKI [6] and with worse outcomes if AKI develops [6,7]. Secondly, use of goaldirected therapy (GDT) strategies for perioperative haemodynamic optimisation have been associated with 
decreased surgical complications $[3,8,9]$ and reduced risk of postoperative AKI [10]. GDT strategies involve the use of intensive monitoring and specific haemodynamic targets to guide early aggressive fluid and/or vasoactive drug administration. While individually persuasive, collectively these two lines of evidence appear difficult to reconcile. On the one hand, fluid overload appears harmful overall, and to the kidney in particular; on the other hand, goal-directed FR reduces mortality, morbidity and renal dysfunction. The evidence base for both of these conclusions is strongest in surgery, and consequently perioperative resuscitation was chosen as the setting to best explore this issue.

This seeming conflict in evidence may be the cause of controversy surrounding the extent of FR in patients at risk of AKI. Yet, without knowledge of the relative timing of onset, duration and quantity of fluid given during GDT-FR compared with the current standard of care, it is impossible to gauge whether the apparent benefits of more conservative FR and those of goal-directed haemodynamic therapy are truly in opposition. Accordingly, we conducted a systematic literature review and metaanalysis of controlled trials of goal-directed resuscitation protocols in the settings of surgery. Our focus was on AKI and the quantity of fluid administered. We hypothesised that the beneficial effects of GDT on subsequent renal function are related to the amount of additional fluid given during GDT compared with control patients, treated according to the standard of care.

\section{Methods}

\section{Aims}

We aimed to identify and review studies describing haemodynamically targeted FR during GDT for perioperative optimisation. In particular, we aimed to determine whether such protocols have a beneficial effect on renal function and to what extent this treatment involves increased fluid administration in comparison with control groups treated according to the standard of care.

\section{Electronic search strategy}

We interrogated the MEDLINE electronic reference database using a combination of search terms (Figure 1). In addition, bibliographies of known systematic reviews and retrieved articles were examined for references of potential relevance. Our wide search terms defined a set of records containing randomised controlled trials (RCTs) of GDT in surgery; these abstracts were then manually examined to identify a subset of studies of potential relevance for manuscript retrieval. These publications were examined manually for accordance with our inclusion criteria. Additional checks were made to ensure none of these publications had been subject to retraction [11].

\section{Study inclusion criteria Population}

We considered only patients undergoing major surgery. Studies involving mixed populations of critically ill patients were excluded.

\section{Intervention}

GDT was defined as monitoring of physiological measurements related to adequacy of cardiac output, systemic oxygen delivery or ventricular preload and providing specific therapy to target normal or supranormal values by FR alone or in combination with inotropic drugs. GDT was required to commence before or within 8 hours from commencement of surgery.

\section{Between-group comparisons}

We considered studies that reported the effects of GDT versus standard haemodynamic therapy, with special emphasis on the comparisons of cumulative volumes of FR. We excluded RCTs with no difference in, or no description of, fluid management strategies between groups, RCTs comparing differing GDT protocols targeting the same physiological goal, or studies without predefined end points. A specific requirement was that studies needed to report a volumetric quantification of FR. We did not consider studies that examined the effect of differing maintenance fluid infusions in our main analysis.

\section{Outcome}

Studies were required to report a renal outcome measure that defined AKI - this could be an absolute or relative change in serum creatinine or urea, a defined period of oliguria, a need for renal replacement therapy for AKI or some combination of these.

\section{Data collection}

We collected data on the study participants, the setting, the timing and duration of the intervention, the definition of AKI used, the method of physiological monitoring and the GDT protocol. We additionally examined indicators of study quality and reporting, including documentation of the method of randomisation, the extent of blinding and description of drop-outs based on the score proposed by Jadad and colleagues [12]. Finally, we documented the recorded volume of fluid administered and whether there were statistical differences in these quantities.

To assess the volumes of fluid administered in the treatment and control groups we calculated the difference in the mean or median total fluid volume delivered between groups. When a total was not reported, we summed the mean or median volumes of crystalloids, colloid and blood products given during the protocol period in the GDT and control groups to estimate the total fluid 


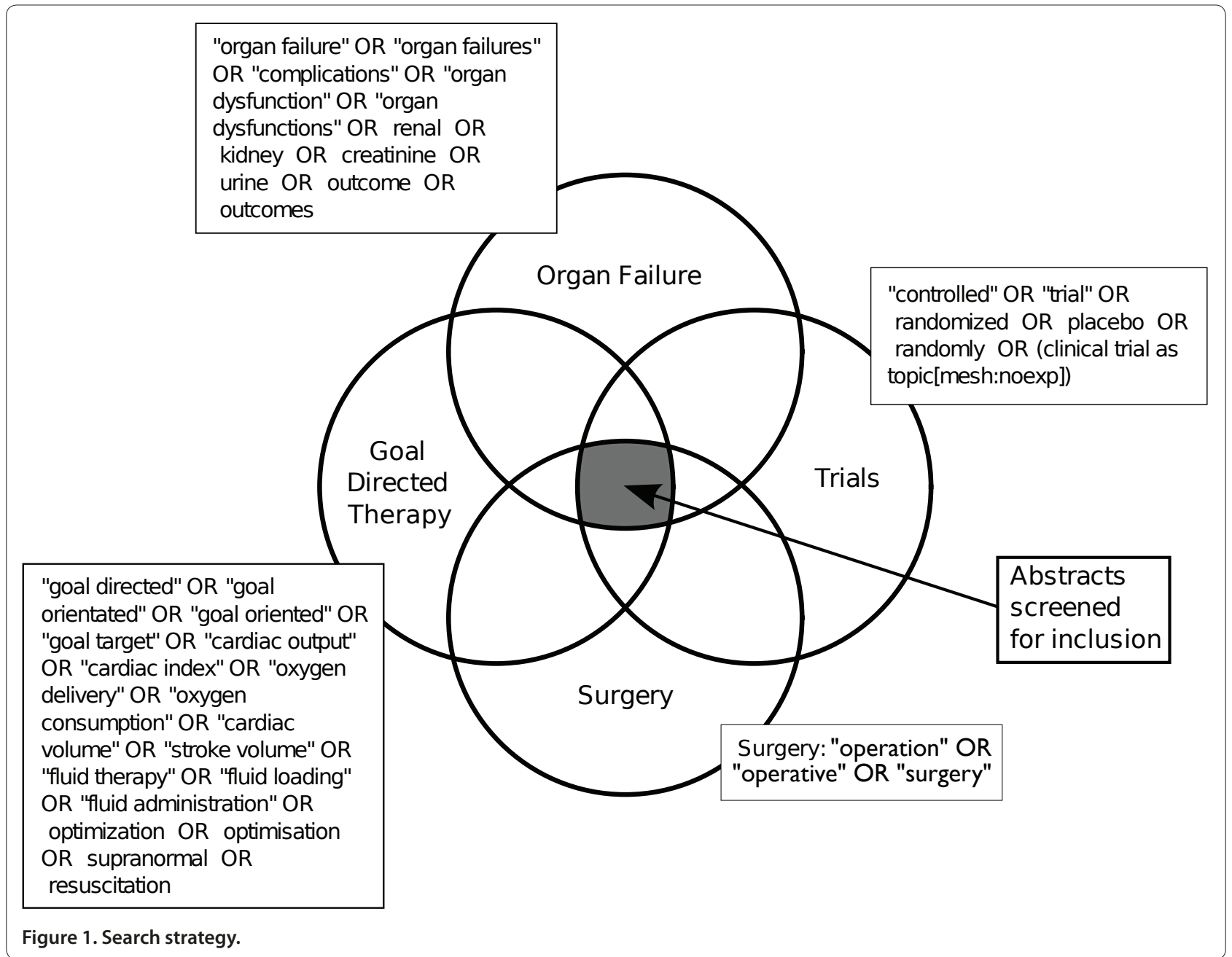

volume delivered in each group. We defined studies employing a larger volume of FR during GDT as those demonstrating a statistically significant difference between one or more types of fluid administered between groups during GDT. In addition, we excluded from our definition any studies where the difference in mean or median fluid administration during the GDT protocol period was $<500 \mathrm{ml}$, an arbitrary definition of a clinically insignificant difference in fluid administration between GDT and standard of care.

\section{Statistical analysis}

Meta-analysis was performed using R: A language and environment for statistical computing (R Foundation for Statistical Computing, Vienna, Austria [13]), utilising the packages meta, metafor and rmeta. As studies compared differing interventions in differing settings with differing measures of renal function, a random effects model was employed for all analyses. The effect of GDT in the metaanalysis of pooled studies was assessed by calculation of the odds ratio (OR) for AKI: OR $<1$ favoured GDT over the control group. The 95\% confidence intervals (CIs) of the ORs and two-sided $P$ values were calculated. A statistical difference favouring or disfavouring GDT was considered to occur if the $95 \%$ CI failed to cross the $\mathrm{OR}=1$ line (equivalent to $P<0.05$ ).

Statistical heterogeneity [14] was assessed by calculation of the $I^{2}$ statistic and the Q-test $[15,16]$. Heterogeneity or inconsistency was considered significant if $I^{2}$ was $>40 \%$ or if the $P$ value for the Q-test was $<0.1$ [17]. When heterogeneity was present we assessed for outlier studies using a Galbraith plot [18] and repeated the analysis excluding the most significant outlier [19]. Our metaanalysis was then repeated with subdivision by difference in volume of FR between the GDT and control groups and by use or nonuse of inotropes in GDT protocols.

\section{Results}

Our search strategy on GDT in surgery identified 3,797 abstracts or articles from which we examined 53 
potentially relevant manuscripts, finding 24 articles that met our inclusion criteria [20-43] (Figure 2). All studies were in adult patients. Among the surgical studies not meeting the inclusion criteria, three recent studies examined a more restrictive perioperative maintenance fluid infusion combined with the use of GDT in one or both groups to facilitate a conservative maintenance fluid regimen [44-46]. While these studies were not directly comparable with standard GDT trials, they are of relevance to a discussion of the interaction between GDT and fluid balance and we elected to examine these investigations in a separate analysis.

The study details, population, size and quality indicators are presented in Table 1; 15 of 24 studies had a Jadad score of 3 . Blinding is inherently difficult when using a treatment intervention triggered by invasive monitoring, and most studies were unblinded or partially blinded; while the randomisation method and dropouts were generally well recorded. As most studies were of similar quality, we did not subdivide our analysis based on quality.

Analysis of details of GDT interventions and renal outcome measures are presented in Table 2 and fluid administration during GDT is presented in Table 3; further details of individual fluids administered in these studies are provided in Additional file 1. Diagnostic criteria for AKI varied widely from a defined increment in serum creatinine from baseline or oliguria over a specified duration to a need for renal replacement therapy (Table 2); for two studies $[37,38]$ renal outcome data not in the original trial publication were reported in a previous meta-analysis [10]. Given the large variation in definition of AKI for studies of GDT in surgery, we performed a further sensitivity analysis considering only studies where AKI was defined as an elevation of creatinine from baseline of $\leq 100 \%$ or an absolute rise in serum creatinine of $\leq 200 \mu \mathrm{mol} / \mathrm{l}$, representing studies using creatinine criteria for AKI thus including patients with less severe postoperative renal dysfunction (approximating RIFLE risk or injury AKI).

\section{Goal-directed therapy in surgery}

Of the 24 included studies, 16 reported a statistically significant greater quantity of colloid, crystalloid or total fluid administered during GDT. In two studies the difference in fluid administration was statistically significant but the total difference in FR between the GDT and control groups during the study period was small $(+241 \mathrm{ml}$ and $+450 \mathrm{ml})$, so these studies were reallocated to the equivalent volume of FR with GDT group [32,39]. Fourteen studies were thus deemed to involve a greater volume of FR during GDT, their median difference in FR between the GDT and control groups being $+1,247 \mathrm{ml}$ (range 500 to $11,099 \mathrm{ml}$ ). In the 10 studies categorised as

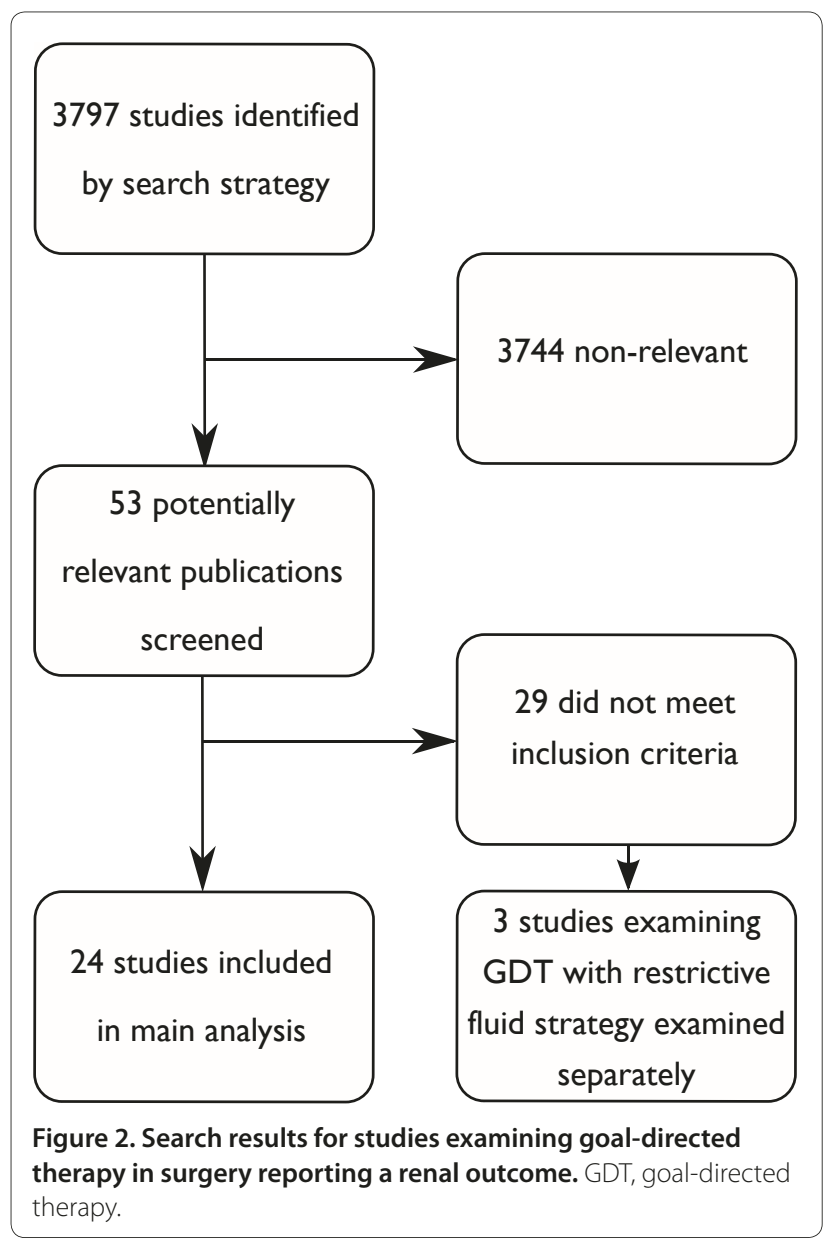

having no greater volume of total FR during GDT, the median difference in FR was $10 \mathrm{ml}$ (range -600 to $+529 \mathrm{ml}$ ). Among all 24 studies, the median difference in FR was $+555 \mathrm{ml}$.

Overall, 13 studies employed inotropic drugs in some or all patients in the GDT group, 10 studies did not use inotropic drugs, and one study had two treatment arms one arm involving infusion of dopexamine in addition to stroke-volume-directed fluid boluses, and the other arm involving stroke-volume-guided therapy alone [31] (Table 2). In the meta-analysis this study was included as a single study in the overall analysis and in the analysis based on fluid therapy, but was treated as two studies when dividing studies on the basis of inotropic use (Figure 3 and Table 4).

Overall, GDT in surgery was associated with a significantly lower incidence of AKI $(\mathrm{OR}=0.59,95 \% \mathrm{CI}=0.39$ to $0.89, P=0.013$; 24 studies, $n=2,763$ patients) (Figure 3 and Table 4). When subdividing by larger versus equivalent fluid administered during GDT, studies involving equivalent overall fluid therapy were associated with a lower incidence of AKI (OR $=0.47,95 \% \mathrm{CI}=0.29$ to 0.76 , 
Table 1. Study settings, size and quality of studies of fluid therapy

\begin{tabular}{|c|c|c|c|c|c|c|c|c|}
\hline Author & Year & Country & Population & Number & Blinded & Randomised & Dropouts & $\begin{array}{l}\text { Jadad } \\
\text { score }\end{array}$ \\
\hline \multicolumn{9}{|l|}{ Studies of GDT in surgery } \\
\hline Challand and colleagues [25] & 2012 & UK & $\begin{array}{l}\text { Major open or laparoscopic } \\
\text { colorectal surgery }\end{array}$ & 179 & 1 & 2 & 1 & 4 \\
\hline Cecconi and colleagues [24] & 2011 & Italy & Elective total hip arthroplasty & 40 & 1 & 2 & 1 & 3 \\
\hline Mayer and colleagues [35] & 2010 & Germany & Major abdominal surgery & 60 & 1 & 2 & 1 & 3 \\
\hline Jhanji and colleagues [31] & 2010 & UK & Major gastrointestinal surgery & 135 & 0 & 2 & 1 & 3 \\
\hline Forget and colleagues [28] & 2010 & Belgium & Major abdominal surgery & 82 & 0 & 1 & 1 & 2 \\
\hline Benes and colleagues [21] & 2010 & Czech Republic & $\begin{array}{l}\text { High-risk elective intra-abdominal } \\
\text { surgery }\end{array}$ & 120 & 1 & 2 & 1 & 4 \\
\hline Harten and colleagues [30] & 2008 & UK - Scotland & $\begin{array}{l}\text { Patients over the age of } 50 \\
\text { undergoing emergency } \\
\text { abdominal surgery }\end{array}$ & 29 & 0 & 2 & 1 & 3 \\
\hline Kapoor and colleagues [32] & 2008 & India & $\begin{array}{l}\text { Patients with EuroSCORE } \\
\geq 3 \text { undergoing coronary } \\
\text { artery bypass surgery under } \\
\text { cardiopulmonary bypass }\end{array}$ & 30 & 0 & 2 & 1 & 3 \\
\hline Donati and colleagues [27] & 2007 & Italy & Major abdominal surgery & 135 & 0 & 2 & 1 & 3 \\
\hline Lopes and colleagues [34] & 2007 & Brazil & High-risk surgery & 33 & 0 & 2 & 1 & 3 \\
\hline Chytra and colleagues [26] & 2006 & Czech Republic & $\begin{array}{l}\text { ICU admissions undergoing } \\
\text { surgery for major trauma with } \\
\text { expected blood loss }>2 \text { I }\end{array}$ & 162 & 0 & 1 & 1 & 2 \\
\hline Wakeling and colleagues [42] & 2006 & UK & Colorectal resection & 128 & 0 & 2 & 1 & 3 \\
\hline Noblett and colleagues [37] & 2006 & UK & Elective colorectal resection & 108 & 2 & 1 & 1 & 4 \\
\hline Pearse and colleagues [38] & 2005 & UK & High-risk general surgical patients & 122 & 0 & 2 & 1 & 3 \\
\hline McKendry and colleagues [36] & 2004 & UK & Cardiac surgery & 174 & 0 & 2 & 1 & 3 \\
\hline Gan and colleagues [29] & 2002 & USA & $\begin{array}{l}\text { Major elective surgery with an } \\
\text { anticipated blood loss }>500 \mathrm{ml}\end{array}$ & 100 & 0 & 2 & 1 & 3 \\
\hline Bonazzi and colleagues [23] & 2002 & Italy & $\begin{array}{l}\text { Infrarenal abdominal aortic } \\
\text { aneurysm repair }\end{array}$ & 100 & 0 & 2 & 0 & 2 \\
\hline Venn and colleagues [41] & 2002 & UK & $\begin{array}{l}\text { Proximal femoral fracture repair, } \\
\text { age }>65\end{array}$ & 90 & 0 & 2 & 1 & 3 \\
\hline Lobo and colleagues [33] & 2000 & Brazil & $\begin{array}{l}\text { Patients }>60 \text { years old and/or with } \\
\text { chronic diseases of vital organs } \\
\text { who underwent major elective } \\
\text { surgery }\end{array}$ & 37 & 0 & 2 & 1 & 3 \\
\hline Pölönen and colleagues [39] & 2000 & Finland & Elective cardiac surgery & 403 & 0 & 2 & 1 & 3 \\
\hline Wilson and colleagues [43] & 1999 & UK & $\begin{array}{l}\text { Patients undergoing major } \\
\text { elective surgery who were at } \\
\text { risk of developing postoperative } \\
\text { complications }\end{array}$ & 138 & 0 & 2 & 1 & 3 \\
\hline Valentine and colleagues [40] & 1998 & USA & Aortic surgery & 120 & 0 & 1 & 1 & 2 \\
\hline Bender and colleagues [20] & 1997 & USA & Elective vascular surgery & 104 & 0 & 1 & 1 & 2 \\
\hline Bishop and colleagues [22] & 1995 & USA & Surgery for major trauma & 125 & 0 & 1 & 1 & 2 \\
\hline \multicolumn{9}{|c|}{ Studies using GDT in surgery comparing restrictive vs. conservative maintenance fluids } \\
\hline Lobo and colleagues [46] & 2011 & Brazil & High-risk elective surgery & 88 & 0 & 1 & 1 & 2 \\
\hline Futier and colleagues [44] & 2011 & France & Major abdominal surgery & 70 & 1 & 2 & 1 & 4 \\
\hline Jammer and colleagues [45] & 2010 & Norway & $\begin{array}{l}\text { Open colorectal and lower } \\
\text { intestinal surgery }\end{array}$ & 241 & 0 & 2 & 1 & 3 \\
\hline
\end{tabular}


Table 2. Goal-directed therapy strategies and renal outcome measures in studies

\begin{tabular}{|c|c|c|c|c|c|c|}
\hline Study & Goal & $\begin{array}{l}\text { Timing of } \\
\text { intervention }\end{array}$ & GDT monitor & Intervention & Renal outcome & $\begin{array}{l}\text { Significantly } \\
\text { larger fluid } \\
\text { in GDT }\end{array}$ \\
\hline \multicolumn{7}{|c|}{ Studies of GDT in surgery } \\
\hline $\begin{array}{l}\text { Challand and } \\
\text { colleagues [25] }\end{array}$ & SV optimisation & Intraoperative & $\begin{array}{l}\text { Oesophageal } \\
\text { Doppler }\end{array}$ & $\begin{array}{l}\text { SV-guided } 200 \mathrm{ml} \\
\text { HES } 6 \% \text { boluses }\end{array}$ & $\begin{array}{l}\text { Creatinine increase to }>149 \% \\
\text { of baseline during first } \\
\text { postoperative week }\end{array}$ & Yes \\
\hline $\begin{array}{l}\text { Cecconi and } \\
\text { colleagues [24] }\end{array}$ & $\begin{array}{l}\text { Maximise SV and } \mathrm{DO}_{2} \mathrm{I} \\
>600 \mathrm{ml} / \mathrm{min} / \mathrm{m}^{2}\end{array}$ & $\begin{array}{l}\text { Intraoperative } \\
\text { and 1-hour } \\
\text { postoperative }\end{array}$ & FloTracNigileo & $\begin{array}{l}\text { SV-guided HES } \\
6 \% \text { boluses } \pm \\
\text { dobutamine }\end{array}$ & Oliguria or AKI & Yes \\
\hline $\begin{array}{l}\text { Mayer and } \\
\text { colleagues [35] }\end{array}$ & $\begin{array}{l}\mathrm{Cl}>2.5 \mathrm{I} / \mathrm{min} / \mathrm{m}^{2}, \mathrm{SV} \\
\text { variation }<12 \%\end{array}$ & Intraoperative & FloTracNigileo & $\begin{array}{l}250 \text { to } 500 \mathrm{ml} \\
\text { colloid boluses } \pm \\
\text { dobutamine }\end{array}$ & $\begin{array}{l}\cup O<500 \mathrm{ml} / \text { day or required } \\
\text { dialysis for acute renal failure }\end{array}$ & No \\
\hline $\begin{array}{l}\text { Jhanji and } \\
\text { colleagues [31] }\end{array}$ & SV optimisation & $\begin{array}{l}\text { For } 8 \text { hours } \\
\text { postoperative }\end{array}$ & Lidco & $\begin{array}{l}\text { Gelatin } 250 \mathrm{ml} \\
\text { to optimise SV } \pm \\
\text { dopexamine } \\
0.5 \mu \mathrm{g} / \mathrm{kg} / \mathrm{min}\end{array}$ & AKIN criteria AKI & No \\
\hline $\begin{array}{l}\text { Forget and } \\
\text { colleagues [28] }\end{array}$ & $\begin{array}{l}\text { Pulse oximeter } \\
\text { plethysmogram variability } \\
\text { index }<13 \%\end{array}$ & Intraoperative & Pulse oximeter & $\begin{array}{l}250 \mathrm{ml} \text { colloid } \\
\text { boluses }\end{array}$ & Postoperative oliguria or RRT & No \\
\hline $\begin{array}{l}\text { Benes and } \\
\text { colleagues [21] }\end{array}$ & $\begin{array}{l}\text { SV variation }<10 \%, \mathrm{Cl} \\
>2.5 \mathrm{I} / \mathrm{min} / \mathrm{m}^{2}\end{array}$ & Intraoperative & FloTracNigileo & $\begin{array}{l}3 \mathrm{ml} / \mathrm{kg} \text { colloid } \\
\text { boluses } \pm \\
\text { dobutamine }\end{array}$ & $\begin{array}{l}\text { AKI by POSSUM scoring } \\
\text { (increase in blood urea } \\
>5 \mathrm{mmol} / \mathrm{l} \text { from preoperative } \\
\text { levels) or RRT }\end{array}$ & No \\
\hline $\begin{array}{l}\text { Harten and } \\
\text { colleagues [30] }\end{array}$ & $\begin{array}{l}\text { Pulse pressure variation } \\
<10 \%\end{array}$ & Intraoperative & Lidco & $\begin{array}{l}\text { Boluses of } 250 \mathrm{ml} \\
\text { of } 6 \% \mathrm{HES} \text { if pulse } \\
\text { pressure variation } \\
>10 \%\end{array}$ & $\begin{array}{l}\cup O<500 \mathrm{ml} / \text { day or increase } \\
\text { in } \mathrm{SCr}>30 \% \text { from the } \\
\text { preoperative level }\end{array}$ & Yes \\
\hline $\begin{array}{l}\text { Kapoor and } \\
\text { colleagues [32] }\end{array}$ & $\begin{array}{l}\mathrm{CVP}>6 \mathrm{mmHg}, \mathrm{SWV}<10 \% \\
\mathrm{Cl} 2.5 \text { to } 4.2 \mathrm{l} / \mathrm{min} / \mathrm{m}^{2} \\
\mathrm{ScVO}_{2}>70 \%\end{array}$ & $\begin{array}{l}\text { For } 8 \text { hours } \\
\text { postoperative }\end{array}$ & FloTrac and $\mathrm{ScvO}_{2}$ & $\begin{array}{l}\text { Colloid boluses } \\
\text { inotropes and blood } \\
\text { per protocol }\end{array}$ & $\begin{array}{l}\text { Increase in } \mathrm{SCr}>150 \mu \mathrm{mol} / \mathrm{l}, \\
\mathrm{UO}<750 \mathrm{ml} / 24 \text { hours }\end{array}$ & $\mathrm{No}^{\mathrm{a}}$ \\
\hline $\begin{array}{l}\text { Donati and } \\
\text { colleagues [27] }\end{array}$ & $\mathrm{O}_{2} \mathrm{ER}<27 \%$ & $\begin{array}{l}\text { Intraoperative to } \\
24 \text { hours }\end{array}$ & CVC, arterial line & $\begin{array}{l}\text { Colloid boluses } \pm \\
\text { dobutamine }\end{array}$ & $\mathrm{SCr}>2 \mathrm{mg} / \mathrm{dl}$ or need for RRT & No \\
\hline $\begin{array}{l}\text { Lopes and } \\
\text { colleagues [34] }\end{array}$ & $\begin{array}{l}\text { Pulse pressure variation } \\
<10 \%\end{array}$ & Intraoperative & Arterial line & $\begin{array}{l}\text { Colloid boluses } 6 \% \\
\text { HES }\end{array}$ & $\begin{array}{l}\cup O<500 \mathrm{ml} / \text { day or serum } \\
\text { creatinine }>170 \mu \mathrm{mol} / \mathrm{l} \text { or } \\
\text { dialysis for AKI }\end{array}$ & Yes \\
\hline $\begin{array}{l}\text { Chytra and } \\
\text { colleagues [26] }\end{array}$ & $\begin{array}{l}\text { SV optimisation with FTc } \\
0.35 \text { to } 0.4 \text { seconds }\end{array}$ & $\begin{array}{l}12 \text { hours } \\
\text { postoperative }\end{array}$ & $\begin{array}{l}\text { Oesophageal } \\
\text { Doppler }\end{array}$ & $\begin{array}{l}\text { Colloid boluses } 250 \\
\text { ml gelatin or } 6 \% \text { HES }\end{array}$ & Need for RRT & Yes \\
\hline $\begin{array}{l}\text { Wakeling and } \\
\text { colleagues [42] }\end{array}$ & SV optimisation & Intraoperative & $\begin{array}{l}\text { Oesophageal } \\
\text { Doppler }\end{array}$ & $\begin{array}{l}\text { Gelatin colloid } \\
\text { boluses }\end{array}$ & $\begin{array}{l}\cup O<500 \mathrm{ml} / \text { day or increase } \\
\text { in } \mathrm{SCr}>30 \% \text { from the } \\
\text { preoperative level }\end{array}$ & Yes \\
\hline $\begin{array}{l}\text { Noblett and } \\
\text { colleagues [37] }\end{array}$ & $\begin{array}{l}\text { SV optimisation with FTc } \\
0.35 \text { to } 0.4 \text { seconds }\end{array}$ & Intraoperative & $\begin{array}{l}\text { Oesophageal } \\
\text { Doppler }\end{array}$ & $\begin{array}{l}6 \% \text { HES boluses } 7 \text { or } \\
3 \mathrm{ml} / \mathrm{kg}\end{array}$ & $\begin{array}{l}\text { Increase in SCr or need for } \\
\text { RRT }\end{array}$ & No \\
\hline $\begin{array}{l}\text { Pearse and } \\
\text { colleagues [38] }\end{array}$ & $\begin{array}{l}\text { Optimise SV and DO I } \\
>600 \mathrm{ml} / \mathrm{min} / \mathrm{m}^{2}\end{array}$ & $\begin{array}{l}\text { Postoperative } \\
8 \text { hours }\end{array}$ & Lidco & $\begin{array}{l}\text { Gelatin boluses } \\
\text { to optimise SV } \pm \\
\text { dopexamine }\end{array}$ & Need for RRT & Yes \\
\hline $\begin{array}{l}\text { McKendry and } \\
\text { colleagues [36] }\end{array}$ & Stroke index $>35 \mathrm{ml} / \mathrm{m}^{2}$ & $\begin{array}{l}\text { Postoperative } \\
4 \text { hours }\end{array}$ & $\begin{array}{l}\text { Oesophageal } \\
\text { Doppler }\end{array}$ & $\begin{array}{l}200 \mathrm{ml} \text { boluses } \\
\text { blood or colloid }\end{array}$ & Need for RRT & Yes \\
\hline $\begin{array}{l}\text { Gan and } \\
\text { colleagues [29] }\end{array}$ & $\begin{array}{l}\text { SV optimisation with FTc } \\
0.35 \text { to } 0.4 \text { seconds }\end{array}$ & Intraoperative & $\begin{array}{l}\text { Oesophageal } \\
\text { Doppler }\end{array}$ & $\begin{array}{l}200 \mathrm{ml} \text { boluses of } \\
6 \% \mathrm{HES}\end{array}$ & $\begin{array}{l}\cup O<500 \mathrm{ml} / \text { day or increase } \\
\text { in } \mathrm{SCr}>30 \% \text { from the } \\
\text { preoperative level }\end{array}$ & Yes \\
\hline $\begin{array}{l}\text { Bonazzi and } \\
\text { colleagues [23] }\end{array}$ & $\begin{array}{l}\mathrm{Cl}>3.0 \mathrm{l} / \mathrm{min} / \mathrm{m}^{2}, \text { PAOP } 10 \\
\text { to } 18 \mathrm{mmHg}, \mathrm{SVR}<1,450 \\
\text { dyne } \times \mathrm{second} / \mathrm{cm}^{5}, \mathrm{DO}_{2} \mathrm{I} \\
>600 \mathrm{ml} / \mathrm{min} / \mathrm{m}^{2}\end{array}$ & $\begin{array}{l}\text { Preoperative } \\
\text { to } 2 \text { days } \\
\text { postoperative }\end{array}$ & $\begin{array}{l}\text { Pulmonary artery } \\
\text { catheter }\end{array}$ & $\begin{array}{l}\text { Fluid, blood, } \\
\text { dobutamine }\end{array}$ & $\begin{array}{l}\text { Oliguria requiring high-dose } \\
\text { frusemide or RRT }\end{array}$ & Yes \\
\hline
\end{tabular}


Table 2. Continued

\begin{tabular}{|c|c|c|c|c|c|c|}
\hline Study & Goal & $\begin{array}{l}\text { Timing of } \\
\text { intervention }\end{array}$ & GDT monitor & Intervention & Renal outcome & $\begin{array}{l}\text { Significantly } \\
\text { larger fluid } \\
\text { in GDT }\end{array}$ \\
\hline \multicolumn{7}{|c|}{ Studies of GDT in surgery (continued) } \\
\hline $\begin{array}{l}\text { Venn and } \\
\text { colleagues [41] }\end{array}$ & $\begin{array}{l}\text { Two GDT groups: CVP } \\
>14 \text { mmHg or SV } \\
\text { optimisation with FTC } \\
>0.35 \text { seconds }\end{array}$ & Intraoperative & $\begin{array}{l}\text { CVC or oesophageal } \\
\text { Doppler }\end{array}$ & $\begin{array}{l}\text { Gelatin boluses } 100 \\
\text { to } 200 \mathrm{ml}\end{array}$ & $\begin{array}{l}\cup O<500 \mathrm{ml} / \text { day or increase } \\
\text { in } \mathrm{SCr}>30 \% \text { from the } \\
\text { preoperative level }\end{array}$ & Yes \\
\hline $\begin{array}{l}\text { Lobo and } \\
\text { colleagues [33] }\end{array}$ & $\mathrm{DO}_{2} \mathrm{I}>600 \mathrm{ml} / \mathrm{min} / \mathrm{m}^{2}$ & $\begin{array}{l}\text { Intraoperative to } \\
24 \text { hours }\end{array}$ & $\begin{array}{l}\text { Pulmonary artery } \\
\text { catheter }\end{array}$ & $\begin{array}{l}\text { Fluids, blood, } \\
\text { inotropes }\end{array}$ & $\begin{array}{l}\mathrm{sCr}>3.5 \mathrm{mg} / \mathrm{dl} \text { or } \mathrm{UO} \\
<500 \mathrm{ml} / 24 \text { hours }\end{array}$ & No \\
\hline $\begin{array}{l}\text { Pölönen and } \\
\text { colleagues [39] }\end{array}$ & $\begin{array}{l}\mathrm{ScvO}_{2}>70 \% \text { lactate } \\
<2 \mathrm{mM}\end{array}$ & $\begin{array}{l}8 \text { hours } \\
\text { postoperative }\end{array}$ & $\begin{array}{l}\text { Pulmonary artery } \\
\text { catheter }\end{array}$ & $\begin{array}{l}\text { Fluids, blood, } \\
\text { inotropes }\end{array}$ & $\begin{array}{l}\text { Increase in } \mathrm{SCr}>1.7 \mathrm{mg} / \mathrm{dl} \text {, } \\
\mathrm{UO}<750 \mathrm{ml} / 24 \text { hours }\end{array}$ & $\mathrm{No}^{\mathrm{a}}$ \\
\hline $\begin{array}{l}\text { Wilson and } \\
\text { colleagues [43] }\end{array}$ & $\begin{array}{l}\mathrm{DO}_{2} \mathrm{I}>600 \mathrm{ml} / \mathrm{min} / \mathrm{m}^{2} \\
\mathrm{PAOP}>12 \mathrm{mmHg}\end{array}$ & $\begin{array}{l}\text { Preoperative to } \\
12 \text { to } 24 \text { hours } \\
\text { postoperative }\end{array}$ & $\begin{array}{l}\text { Pulmonary artery } \\
\text { catheter }\end{array}$ & $\begin{array}{l}\text { Fluids, blood } \\
\text { dopexamine or } \\
\text { adrenaline }\end{array}$ & $\begin{array}{l}\cup O<0.5 \mathrm{ml} / \mathrm{kg} / \text { hour for } \\
>3 \text { hours or } 50 \% \text { rise in } \mathrm{SCr}\end{array}$ & No \\
\hline $\begin{array}{l}\text { Valentine and } \\
\text { colleagues [40] }\end{array}$ & $\begin{array}{l}\mathrm{Cl}>2.8 \mathrm{l} / \mathrm{min} / \mathrm{m}^{2}, \text { PAOP } 8 \\
\text { to } 15 \mathrm{mmHg}, \mathrm{SVR}<1,100 \\
\text { dyne } \times \text { second } / \mathrm{cm}^{5}\end{array}$ & $\begin{array}{l}>14 \text { hours } \\
\text { preoperative }\end{array}$ & $\begin{array}{l}\text { Pulmonary artery } \\
\text { catheter }\end{array}$ & $\begin{array}{l}\text { Fluids, dobutamine, } \\
\text { vasodilators }\end{array}$ & $\begin{array}{l}\text { Need for RRT or oliguria } \\
>24 \text { hours with doubling } \\
\text { of SCr }\end{array}$ & Yes \\
\hline $\begin{array}{l}\text { Bender and } \\
\text { colleagues [20] }\end{array}$ & $\begin{array}{l}\mathrm{Cl}>2.8, \mathrm{PAOP} 8 \text { to } \\
14 \mathrm{mmHg}, \mathrm{SVR}<1,100 \\
\text { dyne } \times \text { second } / \mathrm{cm}^{5}\end{array}$ & $\begin{array}{l}\text { Preoperative } \\
\text { to } 16 \text { hours } \\
\text { postoperative }\end{array}$ & $\begin{array}{l}\text { Pulmonary artery } \\
\text { catheter }\end{array}$ & $\begin{array}{l}\text { Fluids, blood, } \\
\text { dopamine, } \\
\text { vasodilators }\end{array}$ & Increase in $\mathrm{SCr}>1 \mathrm{mg} / \mathrm{dl}$ & Yes \\
\hline $\begin{array}{l}\text { Bishop and } \\
\text { colleagues [22] }\end{array}$ & $\begin{array}{l}\mathrm{Cl}>4.5 \mathrm{l} / \mathrm{min} / \mathrm{m}^{2} \\
\mathrm{DO}_{2} \mathrm{I}>760 \mathrm{ml} / \mathrm{min} / \mathrm{m}^{2}, \\
\mathrm{VO}_{2} \mathrm{I}>166 \mathrm{ml} / \mathrm{min} / \mathrm{m}^{2}\end{array}$ & $\begin{array}{l}\text { Attain goal } \\
\text { within } 24 \text { hours } \\
\text { of admission } \\
\text { and maintain for } \\
48 \text { hours }\end{array}$ & $\begin{array}{l}\text { Pulmonary artery } \\
\text { catheter }\end{array}$ & $\begin{array}{l}\text { Fluids, blood, } \\
\text { dobutamine, } \\
\text { vasodilators }\end{array}$ & $\begin{array}{l}\mathrm{SCr}>2 \mathrm{mg} / \mathrm{dl} \text { or twice } \\
\text { baseline in CKD }\end{array}$ & Yes \\
\hline \multicolumn{7}{|c|}{ Studies using GDT in surgery comparing restrictive vs. conservative maintenance fluids } \\
\hline $\begin{array}{l}\text { Lobo and } \\
\text { colleagues [46] }\end{array}$ & $\mathrm{DO}_{2} \mathrm{I}>600 \mathrm{ml} / \mathrm{min} / \mathrm{m}^{2}$ & $\begin{array}{l}\text { Intraoperative } \\
\text { and } 8 \text { hours } \\
\text { postoperative }\end{array}$ & Lidco & $\begin{array}{l}\text { SV-guided gelatin } \\
\text { bolus } \pm \text { dobutamine } \\
\text { in both groups. } \\
\text { Maintenance fluid } 4 \\
\text { vs. } 12 \mathrm{ml} / \mathrm{kg} / \text { hour }\end{array}$ & $\mathrm{SCr} \times 2$ upper limit of normal & Yes \\
\hline $\begin{array}{l}\text { Futier and } \\
\text { colleagues [44] }\end{array}$ & $\begin{array}{l}\text { Variation in peak aortic } \\
\text { flow velocity }(\triangle \mathrm{PV})<13 \%\end{array}$ & Intraoperative & $\begin{array}{l}\text { Oesophageal } \\
\text { Doppler }\end{array}$ & $\begin{array}{l}\triangle \mathrm{PV} \text {-guided } \\
\text { boluses of } 6 \% \mathrm{HES} \\
\text { in both groups. } \\
\text { Maintenance } \\
\text { crystalloid } 6 \text { vs. } \\
12 \mathrm{ml} / \mathrm{kg} / \mathrm{hour}\end{array}$ & $\begin{array}{l}\cup O<500 \mathrm{ml} / \text { day or increase } \\
\text { in } \mathrm{SCr}>30 \% \text { from the } \\
\text { preoperative level or need for } \\
\text { acute RRT }\end{array}$ & Yes \\
\hline $\begin{array}{l}\text { Jammer and } \\
\text { colleagues [45] }\end{array}$ & $\mathrm{ScVO}_{2}>75 \%$ & Intraoperative & CVC & $\begin{array}{l}\text { Colloid boluses } \\
3 \mathrm{ml} / \mathrm{kg} \text { HES in GDT } \\
\text { group. Maintenance } \\
\text { fluid } 100 \mathrm{ml} / \text { hour in } \\
\text { GDT vs. } 800 \mathrm{ml} / \text { hour } \\
\text { in controls }\end{array}$ & SCr increase $>33 \%$ & Yes \\
\hline
\end{tabular}

Statistically significant greater fluid administration in goal-directed therapy (GDT) deemed clinically insignificant (difference $<500 \mathrm{ml}$ ). AKI, acute kidney injury; AKIN, Acute Kidney Injury Network; Cl, cardiac index; CKD, chronic kidney disease; CVP, central venous pressure; CVC, central venous catheter; $\mathrm{DO}_{2} \mathrm{I}$, oxygen delivery index; FTc, corrected flow time; HES, hydroxyethyl starch; $\mathrm{O}_{2}$ ER, oxygen extraction ratio; PAOP, pulmonary artery occlusion pressure; POSSUM, Physiological and Operative Severity Score for the Enumeration of Mortality and Morbidity; RRT, renal replacement therapy; $\mathrm{sCr}$, serum creatinine; $\mathrm{ScvO}_{2}$, central venous oxygen saturation; SV, stroke volume; SVR, stroke volume ratio; SVV, stroke volume variation; UO, urine output; $\mathrm{VO}_{2} \mathrm{l}$, oxygen consumption index. FloTrac, Edwards Lifesciences, Irvine, CA, USA; Lidco, LiDCO Group, London, UK; Vigileo, Edwards Lifesciences, Irvine, CA, USA. aStatistically, but not clinically significantly larger FR in GDT group ( $<500 \mathrm{ml})$.

$P=0.0049 ; 10$ studies, $n=1,268$ patients) (Figure 3 and Table 4) whereas studies categorised as involving larger fluid administration during GDT were not associated with lower incidence $(\mathrm{OR}=0.70,95 \% \mathrm{CI}=0.35$ to 1.41 , $P=0.32$; 13 studies, $n=1,462$ patients); one study was excluded for heterogeneity [34].
Goal-directed therapy and inotropic drug use in surgery Inotropic drug use in GDT patients was associated with a reduction in the incidence of postoperative AKI ( $\mathrm{OR}=0.52,95 \% \mathrm{CI}=0.34$ to $0.80, P=0.003 ; 14$ studies, $n=1,634$ patients) (Figure 3 and Table 4) compared with studies not involving inotropic drugs $(\mathrm{OR}=0.75,95 \%$ 
Table 3. Total fluid administration in studies of perioperative goal-directed fluid therapy

\begin{tabular}{|c|c|c|c|c|c|}
\hline \multirow[b]{2}{*}{ Study } & \multirow[b]{2}{*}{ Year } & \multirow[b]{2}{*}{ Intervention duration } & \multicolumn{2}{|c|}{$\begin{array}{l}\text { Total fluid resuscitation } \\
\text { during intervention (ml) }\end{array}$} & \multirow{2}{*}{$\begin{array}{l}\text { Difference in mean } \\
\text { or median fluid } \\
\text { resuscitation (ml) }\end{array}$} \\
\hline & & & GDT & Control & \\
\hline \multicolumn{6}{|l|}{ Studies of GDT in surgery } \\
\hline Challand and colleagues [25] & 2012 & Operation & 5,278 & 4,046 & 1,232 \\
\hline Cecconi and colleagues [24] & 2011 & Operation +1 hour & 6,032 & 2,635 & 3,397 \\
\hline Mayer and colleagues [35] & 2010 & Operation & 4,528 & 4,494 & 34 \\
\hline Jhanji and colleagues [31] without dopexamine & 2010 & 8 hours & 4,892 & 4,351 & 541 \\
\hline \multicolumn{3}{|l|}{ Jhanji and colleagues [31] with dopexamine } & \multicolumn{2}{|l|}{4,868} & 517 \\
\hline Forget and colleagues [28] & 2010 & Operation & 2,394 & 2,918 & -524 \\
\hline Benes and colleagues [21] & 2010 & Operation & 3,746 & 3,729 & 17 \\
\hline Harten and colleagues [30] & 2008 & Operation & 3,500 & 3,000 & 500 \\
\hline Kapoor and colleagues [32] & 2008 & Operation +8 hours & $330^{\mathrm{a}}$ & $80^{\mathrm{a}}$ & $250^{\mathrm{b}}$ \\
\hline Donati and colleagues [27] & 2007 & Operation +24 hours & 4,391 & 4,285 & 106 \\
\hline Lopes and colleagues [34] & 2007 & Operation & 4,618 & 1,694 & 2,924 \\
\hline Chytra and colleagues [26] & 2006 & 8 hours & 4,516 & 3,649 & 867 \\
\hline Wakeling and colleagues [42] & 2006 & Operation & 5,000 & 4,500 & 500 \\
\hline Noblett and colleagues [37] & 2006 & Operation & 3,638 & 3,834 & -196 \\
\hline Pearse and colleagues [38] & 2005 & 8 hours & 2,962 & 2,164 & 798 \\
\hline McKendry and colleagues [36] & 2004 & 4 hours & 2,020 & 681 & 1,339 \\
\hline Gan and colleagues [29] & 2002 & Operation & 5,420 & 4,775 & 645 \\
\hline Bonazzi and colleagues [23] & 2002 & Operation to postoperative day 2 & 11,130 & 9,130 & 2,000 \\
\hline Venn and colleagues [41] & 2002 & Operation & 2,655 & 2,075 & 580 \\
\hline Lobo and colleagues [33] & 2000 & 24 hours & 6,600 & 7,200 & -600 \\
\hline Pölönen and colleagues [39] & 2000 & 8 hours & 3,193 & 2,772 & $421^{b}$ \\
\hline Wilson and colleagues [43] & 1999 & Operation +12 hours & 3,200 & 3,500 & -300 \\
\hline Valentine and colleagues [40] & 1998 & 14 hours preoperative + operation & 8,650 & 6,710 & 1,940 \\
\hline Bender and colleagues [20] & 1997 & Operation +16 hours & 5,137 & 3,789 & 1,348 \\
\hline Bishop and colleagues [22] & 1995 & Preoperative and intraoperative & $21,975^{c}$ & $10,876^{c}$ & 11,099 \\
\hline \multicolumn{6}{|c|}{ Studies using GDT in surgery comparing restrictive vs. conservative maintenance fluid } \\
\hline Lobo and colleagues [46] & 2011 & Operation +8 hours & 3,517 & 5,250 & $-1,733$ \\
\hline Futier and colleagues [44] & 2011 & Operation & 3,380 & 5,588 & $-2,208$ \\
\hline Jammer and colleagues [45] & 2010 & Operation & 3,869 & 6,491 & $-2,622$ \\
\hline
\end{tabular}

Approximate mean or median fluid volume per patient was approximated from data provided in individual studies (see Addition File 1 for further information). Any statistically significantly greater administration of a component of fluid resuscitation in goal-directed therapy (GDT) over the control group is highlighted in bold. a Extra volume above maintenance fluids $500 \mathrm{ml} / \mathrm{m}^{2} /$ day documented. ${ }^{b}$ Statistically significant greater fluid administration in GDT deemed clinically insignificant (difference $<500 \mathrm{ml}$ ). ' Cumulative fluid balance in study through to end of surgery.

$\mathrm{CI}=0.37$ to $1.53, P=0.43 ; 10$ studies, $n=1,016$ patients); one study was excluded for heterogeneity [34] (Figure 3 and Table 4). The lowest incidence of postoperative AKI occurred in patients with equivalent total fluid delivery in a protocol involving inotropic drugs $(\mathrm{OR}=0.46,95 \%$ $\mathrm{CI}=0.27$ to $0.76, P=0.0036$; eight studies, $n=1,033$ patients) (Figure 3 and Table 4); however, only a small number of studies (three studies, 280 patients) without use of inotropic drugs had equivalent total fluid delivery in the treatment group, so the effect in this group was difficult to assess.

\section{Goal-directed therapy involving restrictive fluid strategies in surgery}

Three studies involving GDT and more restrictive fluid management were identified (Table 1). Two of these studies involved GDT in both groups with differing maintenance fluid rates during surgery, while one study 


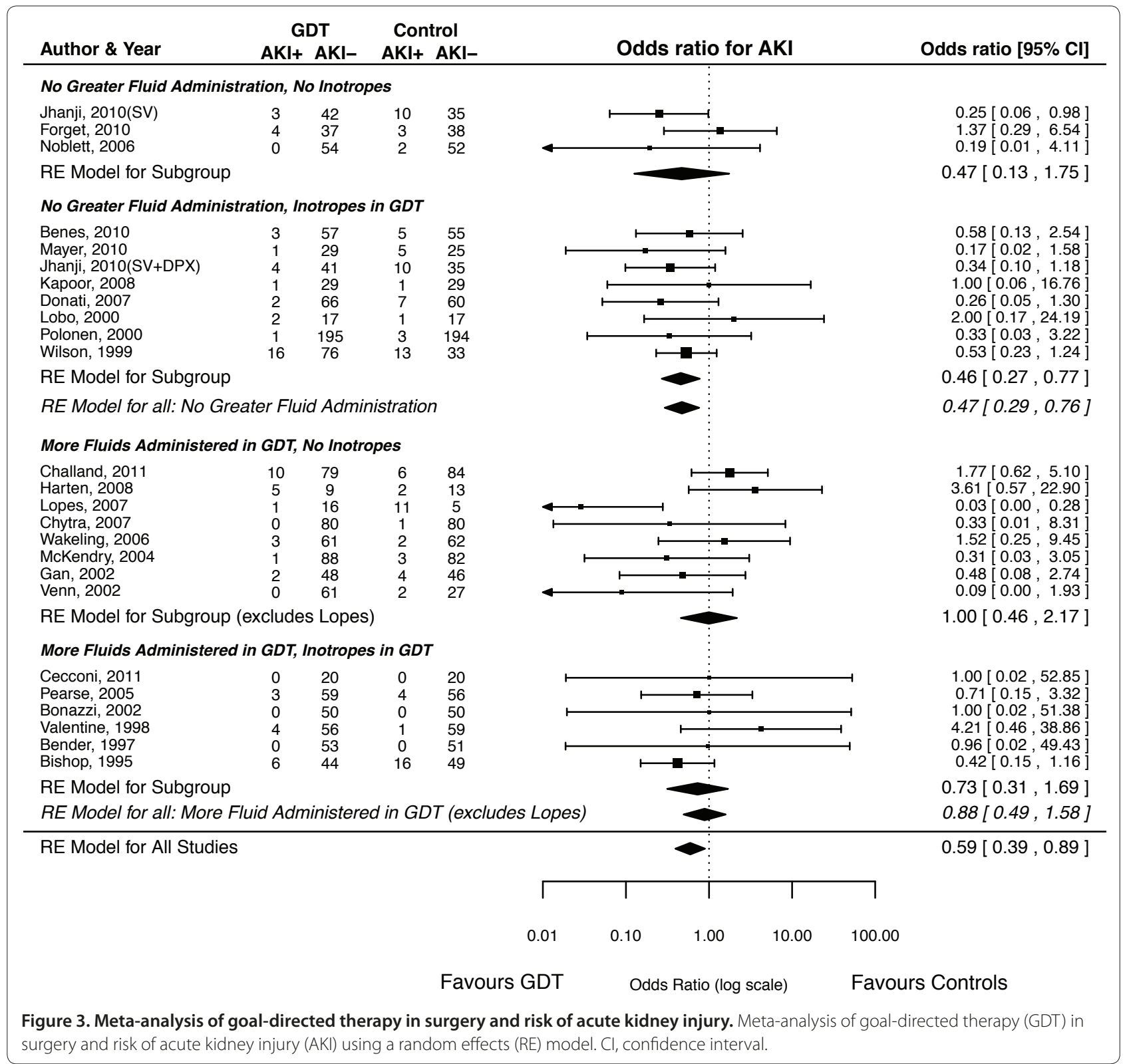

involved GDT only in the more restrictive fluid infusion group (Table 2). The median difference in fluid delivered was $2,208 \mathrm{ml}$ lower (range $-1,733$ to $-2,622 \mathrm{ml}$ ) in the treatment group (Table 3 ). In these three studies there was no clear statistical association between the volume of fluid therapy delivered and AKI (OR for AKI in treatment group $=1.68,95 \% \mathrm{CI}=0.69$ to $4.12, P=0.26$ ) (Figure 4 and Table 4).

\section{Goal-directed therapy in surgery: sensitivity analysis}

We identified 13 studies that used more sensitive and comparable creatinine-based definitions of AKI [20,22,
$25,27,29-32,34,39,41-43]$, one of which [34] was excluded from meta-analyses due to heterogeneity. In this set of trials the association between GDT and protection for AKI was just nonsignificant $(\mathrm{OR}=0.61,95 \% \mathrm{CI}=0.36$ to $1.02, P=0.006$; 12 studies, $n=1,606$ patients) (Table 4 ). However, the risk of AKI was significantly lower in studies with no significant difference in total fluid administration $(\mathrm{OR}=0.41,95 \% \mathrm{CI}=0.23$ to 0.71 , $P=0.002$; five studies, $n=861$ patients) (Table 4 ), while no difference was observed in studies with greater total fluid administration in GDT $(\mathrm{OR}=0.90,95 \% \mathrm{CI}=0.41$ to $1.97, P=0.80$; seven studies, $n=745$ patients) (Table 4 ). 
Table 4. Meta-analysis for prediction of acute kidney injury with measures of heterogeneity

\begin{tabular}{|c|c|c|c|c|c|c|c|}
\hline Comparison & $\begin{array}{l}\text { Number of } \\
\text { studies }\end{array}$ & $\begin{array}{l}\text { Odds ratio } \\
(95 \% \mathrm{Cl})\end{array}$ & $P$ & $\tau^{2}$ & $\begin{array}{l}\text { Q-statistic } \\
P \text { value }\end{array}$ & $I^{2}(\%)$ & $H^{2}$ \\
\hline All studies conventional fluid strategies & 24 & 0.59 (0.39 to 0.89$)$ & 0.013 & 0.19 & 0.21 & 20.2 & 1.25 \\
\hline $\begin{array}{l}\text { GDT involved no clinically significantly } \\
\text { greater fluid administration }\end{array}$ & 10 & 0.47 (0.29 to 0.76$)$ & 0.0049 & 0 & 0.73 & 0 & 1.0 \\
\hline $\begin{array}{l}\text { Clinically significantly more fluids } \\
\text { administered with GDT }\end{array}$ & 14 & 0.70 (0.35 to 1.41$)$ & 0.32 & 0.60 & 0.09 & 39.4 & 1.65 \\
\hline $\begin{array}{l}\text { Clinically significantly more fluids } \\
\text { administered with GDT excluding outlier study }\end{array}$ & 13 & 0.88 (0.49 to 1.58 ) & 0.68 & 0.18 & 0.44 & 16.7 & 1.19 \\
\hline $\begin{array}{l}\text { All studies conventional fluid strategies, } \\
\text { no inotropes }\end{array}$ & $11^{\mathrm{a}}$ & 0.55 (0.24 to 1.26$)$ & 0.16 & 0.97 & 0.03 & 52.5 & 2.11 \\
\hline $\begin{array}{l}\text { All studies conventional fluid strategies, } \\
\text { no inotropes excluding outlier study }\end{array}$ & $10^{\mathrm{a}}$ & 0.75 (0.37 to 1.53$)$ & 0.43 & 0.38 & 0.19 & 30.8 & 1.45 \\
\hline $\begin{array}{l}\text { All studies conventional fluid strategies - } \\
\text { inotropes in GDT }\end{array}$ & $14^{\mathrm{a}}$ & 0.52 (0.34 to 0.80$)$ & 0.0030 & 0 & 0.87 & 0 & 1.0 \\
\hline $\begin{array}{l}\text { No clinically significant greater fluid } \\
\text { administration, no inotropes }\end{array}$ & $3^{\mathrm{a}}$ & $0.47(0.13$ to 1.75$)$ & 0.25 & 0.51 & 0.23 & 37.3 & 1.59 \\
\hline $\begin{array}{l}\text { No clinically significant greater fluid } \\
\text { administration, inotropes in GDT }\end{array}$ & $8^{a}$ & 0.46 (0.27 to 0.77$)$ & 0.0036 & 0 & 0.85 & 0 & 1.0 \\
\hline $\begin{array}{l}\text { Clinically significant more fluids administered } \\
\text { with GDT, no inotropes }\end{array}$ & 8 & 0.55 (0.18 to 1.68$)$ & 0.30 & 1.46 & 0.02 & 60.5 & 2.53 \\
\hline $\begin{array}{l}\text { Clinically significant more fluids administered } \\
\text { with GDT, no inotropes excluding outlier study }\end{array}$ & 7 & 1.0 (0.46 to 2.17$)$ & 0.99 & 0.19 & 0.26 & 17.2 & 1.21 \\
\hline $\begin{array}{l}\text { Clinically significant more fluids administered } \\
\text { with GDT, inotropes in GDT }\end{array}$ & 6 & 0.73 (0.31 to 1.69 ) & 0.46 & 0.11 & 0.61 & 8.9 & 1.1 \\
\hline $\begin{array}{l}\text { Creatinine-based definition of AKI excluding } \\
\text { outlier study }\end{array}$ & 12 & 0.61 (0.36 to 1.02 ) & 0.06 & 0.20 & 0.23 & 26.6 & 1.36 \\
\hline $\begin{array}{l}\text { Creatinine-based definition of AKI, GDT } \\
\text { involved no clinically significantly greater fluid } \\
\text { administration excluding outlier study }\end{array}$ & 5 & 0.41 (0.23 to 0.72 ) & 0.002 & 0 & 0.83 & 0 & 1.0 \\
\hline $\begin{array}{l}\text { Creatinine-based definition of AKI, clinically } \\
\text { significantly more fluids administered with GDT } \\
\text { excluding outlier study }\end{array}$ & 7 & 0.90 (0.41 to 1.97 ) & 0.80 & 0.35 & 0.18 & 33.3 & 1.5 \\
\hline $\begin{array}{l}\text { GDT to assist restrictive fluid strategy in } \\
\text { treatment arm }\end{array}$ & 3 & 1.68 (0.69 to 4.12 ) & 0.26 & 0 & 0.29 & 0 & 1.0 \\
\hline
\end{tabular}

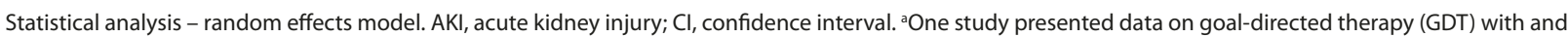
without inotropic therapy [31]. Data from these groups were considered as separate studies, dividing on the basis of use/nonuse of inotropes in GDT.

\section{Discussion}

\section{Key findings}

In this review we have examined the evidence for the use of FR in improving renal outcomes during major surgery. We found that, on average, protocolised GDT did not result in significantly larger volumes of fluid therapy being administered when compared with standard management. Furthermore, most of the protection from AKI seemed to occur in studies reporting equivalent total quantity of fluid administration between groups. From this evidence, it is difficult to ascribe benefits of GDT simply to the provision of greater volumes of intravenous fluids to hypovolaemic patients, suggesting any effect actually derived from other aspects of care. Our findings were similar when only studies using more sensitive creatinine-based definitions of AKI were examined in a sensitivity analysis. Use of inotropes seemed to be associated with lower incidence of AKI with GDT and with the delivery of equivalent amounts of FR.

\section{Relationship to previous studies}

Conventionally, a reduction in RBF and renal ischaemia have been regarded central to the pathogenesis of AKI in critical illness [47]; however, the situation is likely to be more complex [48]. In animal models, AKI has been reported when cardiac output was preserved or elevated [49], and subtotal (90\%) occlusion of the renal arteries did not lead to prolonged AKI [50]. Furthermore, cardiac arrest and warm renal ischaemia in humans only lead to significant AKI in the presence of severe post-resuscitation disease and cardiogenic shock [51]. These observations infer that local and systemic inflammatory responses, 


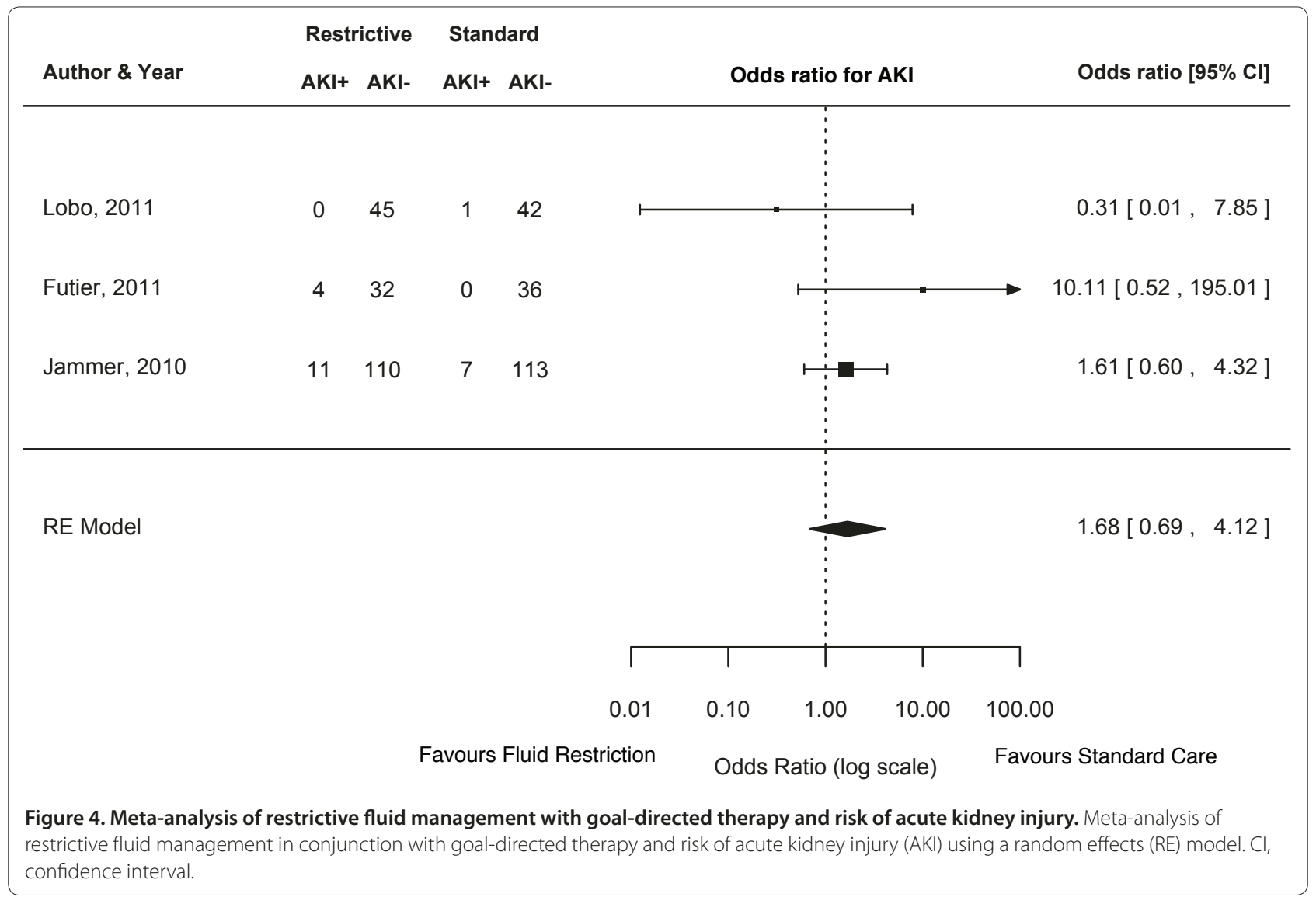

changes in intra-RBF distribution, microcirculatory dysfunction and glomerular haemodynamics may all account for loss of kidney function, even in the presence of maintained or increased RBF.

Fluid administration has been shown to augment both cardiac output and RBF without an overall increase in renal oxygen delivery [52], while FR in an experimental model of haemorrhagic shock can restore blood pressure and cardiac output without recovery of renal tissue oxygen tension [53]. Finally, even when renal oxygen delivery is reduced, increased oxygen extraction may maintain renal oxygen consumption [54] - suggesting that increasing renal oxygen delivery by increasing cardiac output may not avert AKI.

Measurements of cardiac output are rarely made until critical illness is established, RBF measurements are not clinically available, and fluid therapy is routinely targeted to the treatment of arterial hypotension. Absolute or relative hypotension is considered a significant risk factor for the development of AKI [55,56], and lower blood pressure has been associated with the progression of AKI in sepsis [57]. However, hypotension may be related to low cardiac output, systemic vasodilation or a combination of both; and in the setting of systemic hypotension,
RBF may be reduced, elevated or unchanged. As hypotension will only respond to FR if a meaningful increase in cardiac output can be obtained, this response may only be partial or transient in the context of systemic vasodilatation. The RBF effect of FR for hypotension might thus be significant or negligible depending on the context. Bedside physiological measurements such as blood pressure and urine output will be even more indirectly related to the pathogenesis of AKI, although they are routine triggers for FR.

\section{Significance of study findings}

Any potential benefits of FR must be weighed against the consequences of fluid accumulation. In acute illness, exogenous fluid replacement almost inevitably leads to positive fluid balances. Additionally, the critically ill are particularly susceptible to fluid overload [5]. Physiologically, fluid overload results in tissue oedema that may contribute to progressive organ dysfunction. Liberal perioperative fluid strategies, involving large positive fluid balances, have been associated with increased complications after surgery $[3,4,58]$. Importantly, as an encapsulated organ the kidney is itself particularly affected by fluid congestion, because raised venous and intra-capsular 
pressures cause significant decreases in RBF and the glomerular filtration rate [59]. Studies examining liberal versus more conservative fluid management during surgery have failed to demonstrate an increase in AKI in patients receiving less fluid. Instead, recent analysis of results from the Fluid and Catheter Treatment Trial has shown that pursuing an even fluid balance strategy in patients with acute lung injury was associated with a lower incidence of AKI after allowing for the dilutional effect of positive fluid balances on serum creatinine concentration [60].

GDT may thus be beneficial not only by providing extra fluid when it is specifically indicated, but also by allowing earlier and guided use of fluids while preventing the delivery of unnecessary fluid when specific and objective haemodynamic targets are met. Seen in this light, appropriate use of GDT is in harmony, rather than in conflict, with more restrictive approaches to perioperative fluid therapy.

We identified three studies that fell outside the criteria for inclusion in our main analysis where GDT was used in conjunction with fluid restriction [44-46]. These studies moved beyond fluid optimisation using GDT to actively minimise perioperative maintenance fluid administration, resulting in significantly smaller fluid administration than standard care (Table 3). No clear association was found between postoperative AKI and more restrictive fluid balances. A possible trend toward increased incidence of AKI with fluid restriction was seen, but there was significant heterogeneity between a small number of studies. In addition, relative haemodilution in control groups might be expected to artefactually lower the incidence of AKI, which was defined by small rises in serum creatinine. Given that avoidance of fluid overload may improve wider postoperative outcomes, and that GDT appears to protect against AKI when compared with conventional fluid management, the use of GDT strategies to facilitate more restrictive perioperative fluid therapy while avoiding covert hypovolaemia merits further examination in larger trials.

Low-dose inotropes have been associated with better postoperative outcomes when used in perioperative GDT [61]. In our meta-analysis, use of inotropes in GDT was associated with a decreased risk of postoperative AKI - a finding described previously [10]. Without specific trials, however, it is difficult to dissect the relative contributions of inotropy and fluid management on renal outcomes. We found that trials using inotropes in GDT and similar volumes of fluid therapy to controls were associated with the best renal outcomes, while excess fluid and no inotropes were associated with the worst renal outcomes. It is difficult to assess the relative contributions of FR and inotropes, but a synergistic effect is likely; so that goaldirected FR may allow optimal use of inotropic drugs, while inotropic drugs may maximise the haemodynamic effect of fluids and reduce the risk of fluid overload. Notably, an important physiological consequence of increased cardiac contractility is a decrease in right atrial pressure, potentially a beneficial effect limiting the increase in venous pressure with FR.

Are these findings generalisable beyond the context of major surgery? Sepsis is the leading cause of AKI in the ICU and shares many features with systemic inflammatory response after major surgery. In septic shock, even though patients tend to have high cardiac outputs, insensible or distributive fluid losses are common and FR may be required acutely. However, increased capillary permeability makes septic patients particularly prone to generalised tissue oedema and the adverse effects of fluid overload. Historically, approaches targeting supranormal levels of cardiac output and oxygen delivery in patients with established critical illness (including many patients with severe sepsis) have been nonbeneficial [62] or harmful [63]. More recently, treatment protocols immediately after presentation have demonstrated that targeting central venous oxygen saturation may improve survival in septic shock [64]. However, the specific effect of goal-directed resuscitation on renal function was not explicitly examined.

Applying a similar search strategy to that employed in the present systematic review to locate studies examining GDT for the acute treatment of septic shock, we only identified two articles documenting a renal outcome $[65,66]$ - only one of which was in adult patients, precluding a useful meta-analysis in this population. Both of these studies showed a beneficial effect of GDT on renal as well as other outcomes. Significantly, in both studies GDT was associated with earlier FR in at a higher flow rate than standard care, but the overall quantity of FR did not differ between GDT and standard care - suggesting that, in septic shock, early, targeted, therapy may restore haemodynamics and spare later fluid requirements. The same effect - early GDT actually being associated with similar fluid balances to controls over a longer period of treatment - was also observed by Rivers and colleagues in their landmark, although controversial, single-centre study of early GDT in septic shock [64]. By contrast, in the recent FEAST study of fluid management in children presenting with severe sepsis in Kenya, Tanzania and Uganda, rapid fluid bolus volume expansion was associated with significant harm [67]; importantly, however, fluid therapy in the FEAST study was not guided by any form of invasive haemodynamic monitoring. Therefore, in the setting of septic shock as well as surgery, goal-directed haemodynamic resuscitation may be beneficial to the kidney and other organs by targeting FR only to those patients that require it, and avoiding FR in those that do not. At present, however, evidence 
to guide choices in FR for septic shock remains very limited.

\section{Strengths and limitations of review findings}

The meta-analyses performed in this review provide a basis for discussion of the benefits of FR on renal function in the critically ill. Our search was confined to the Pubmed electronic database and it is possible that relevant studies were missed; however, similar metaanalyses with a wider database search identified a similar set of publications $[8,10]$, while inclusion in Pubmed publications should ensure original studies were subject to independent peer review. Studies varied widely in populations, outcome measures and methodology, and conclusions from these data can only be regarded as hypothesis forming - although our results were essentially unchanged when considering only studies with more similar definitions of AKI. GDT protocols are complex and it is difficult to attribute benefit to specific components of each strategy. We can hypothesise that GDT results in timelier FR for patients who require it, even if the total volume of FR within a trial does not differ from the standard of care. While this is probably true considering the nature of GDT, data provided in the surgical trials we considered generally did not include timing of fluids within the study period, so we had no capacity to examine this important question in our analysis.

We did not assess the formulation of intravenous fluids in these studies. This is a complex issue of considerable clinical significance. However, none of the studies we examined set out to specifically compare differing formulations of intravenous fluid, and a variety of intravenous fluids were used in the treatment and control groups in these studies. Individual patient data would be required to explore the effect of fluid formulation on renal outcomes in these studies. It is of course possible that higher volume fluid administration is associated with a larger volume of colloid exposure, potentially associated with harm to the kidney countering the benefits of GDT, but assessing the size of any effect will be very difficult. Major multicentre randomised trials comparing the effect of colloid versus crystalloids for FR in intensive care are nearing publication $[68,69]$ and high-quality data regarding the formulation of FR and outcomes should shortly be available. When these data are available we will be in a much better position to assess the interaction between the volume of FR, the composition of intravenous fluids and AKI.

Our assessments of overall fluid volumes administered between groups in these studies are based on the mean or median values provided in the articles; they are therefore imprecise and do not provide information on the fluid balance, timing of fluid administration or rate of fluid administration. However, even though the exact fluid quantities and their clinical significance could be argued, we feel the general conclusion that perioperative GDT does not, overall, result in much additional intravenous fluid being administered is supported by the studies identified. We considered fluid administration rather than fluid balance, because fluid balances during GDT were only reported in a small number of studies. Three large multicentre RCTs examining the effect of GDT in sepsis (ProCESS [70], ARISE [71] and ProMISe [72]) and another RCT examining perioperative GDT in high-risk surgery (OPTIMISE [73]) are ongoing. Our level of evidence to guide fluid therapy in complex, acute unwell patients should therefore increase in the next 3 to 4 years. In the interim, current evidence suggests that fluid management that rapidly recognises and treats hypovolaemia while avoiding inappropriate fluid loading may be optimal for renal function and wider clinical outcomes.

\section{Conclusions}

Goal-directed haemodynamic therapies involving guided administration of intravenous fluids are associated with decreased incidence of AKI in the setting of major surgery. Evidence in sepsis is presently inconclusive. Crucially, the benefit of these targeted haemodynamic interventions does not seem to be dependent on the amount of fluid administered. On the contrary, GDT seems to achieve better kidney protection when delivering equivalent amounts of FR, perhaps by providing fluid earlier, faster and with the support of inotropic drugs, thus minimizing the harmful effects of fluid overload.

\section{Additional files}

Additional file 1. A table presenting details of fluid administration in studies of goal-directed fluid therapy.

\section{Abbreviations}

AKI, acute kidney injury; Cl, confidence interval; FR, fluid resuscitation; GDT, goal-directed therapy; $O R$, odds ratio; $R B F$, renal blood flow; $R C T$, randomised controlled trial; RIFLE, risk, injury, failure, loss, and end stage.

\section{Competing interests}

The authors declare that they have no competing interests.

\section{Author details}

'Adult Critical Care Unit, The Royal London Hospital, Barts Health NHS Trust, Whitechapel Road, London E1 1BB, UK. ²Division of Nephrology, University Medicine Cluster, National University Health System, 5 Lower Kentridge Road, Singapore 119074. 'Division of Critical Care Medicine, Faculty of Medicine and Dentistry, University of Alberta, 3C1.12 Walter C. Mackenzie Centre, 8440-122 Street, Edmonton, AB, T6G 2B7, Canada. ${ }^{4}$ Department of Intensive Care, Austin Health, 145 Studley Road, Heidelberg, Victoria 3084, Australia.

Published: 7 August 2012 


\section{References}

1. Chertow GM, Burdick E, Honour M, Bonventre JV, Bates DW: Acute kidney injury, mortality, length of stay, and costs in hospitalized patients. J Am SoC Nephrol 2005, 16:3365-3370.

2. Uchino S, Kellum JA, Bellomo R, Doig GS, Morimatsu H, Morgera S, Schetz M, Tan I, Bouman C, Macedo E, Gibney N, Tolwani A, Ronco C, Beginning and Ending Supportive Therapy for the Kidney (BEST Kidney) Investigators: Acute renal failure in critically ill patients: a multinational, multicenter study. JAMA 2005, 294:813-818.

3. Rahbari NN, Zimmermann JB, Schmidt T, Koch M, Weigand MA, Weitz J: Metaanalysis of standard, restrictive and supplemental fluid administration in colorectal surgery. Br J Surg 2009, 96:331-341.

4. Brandstrup B, Tønnesen H, Beier-Holgersen R, Hjortsø E, Ørding H, LindorffLarsen K, Rasmussen MS, Lanng C, Wallin L, Iversen LH, Gramkow CS, Okholm M, Blemmer T, Svendsen P-E, Rottensten HH, Thage B, Riis J, Jeppesen IS, Teilum D, Christensen AM, Graungaard B, Pott F, Danish Study Group on Perioperative Fluid Therapy: Effects of intravenous fluid restriction on postoperative complications: comparison of two perioperative fluid regimens: a randomized assessor-blinded multicenter trial. Ann Surg 2003, 238:641-648.

5. Prowle JR, Echeverri JE, Ligabo EV, Ronco C, Bellomo R: Fluid balance and acute kidney injury. Nat Rev Nephrol 2010, 6:107-115.

6. Payen D, de Pont AC, Sakr Y, Spies C, Reinhart K, Vincent JL; Sepsis Occurrence in Acutely III Patients Investigators: A positive fluid balance is associated with a worse outcome in patients with acute renal failure. Crit Care 2008, $12: R 74$.

7. Bouchard J, Soroko SB, Chertow GM, Himmelfarb J, Ikizler TA, Paganini EP, Mehta RL: Fluid accumulation, survival and recovery of kidney function in critically ill patients with acute kidney injury. Kidney Int 2009, 76:422-427.

8. Dalfino L, Giglio MT, Puntillo F, Marucci M, Brienza N: Haemodynamic goaldirected therapy and postoperative infections: earlier is better. a systematic review and meta-analysis. Crit Care 2011, 15:R154.

9. Giglio MT, Marucci M, Testini M, Brienza N: Goal-directed haemodynamic therapy and gastrointestinal complications in major surgery: a metaanalysis of randomized controlled trials. Br J Anaesth 2009, 103:637-646.

10. Brienza N, Giglio MT, Marucci M, Fiore T: Does perioperative hemodynamic optimization protect renal function in surgical patients? A meta-analytic study. Crit Care Med 2009, 37:2079-2090.

11. Editors-in-Chief Statement Regarding Published Clinical Trials Conducted without IRB Approval by Joachim Boldt [http://www.bja.ac.uk/wp-content/ uploads/2011/02/EIC-Joint-Statement-on-Retractions.4Mar2011.pdf]

12. Jadad AR, Moore RA, Carroll D, Jenkinson C, Reynolds DJ, Gavaghan DJ, McQuay HJ: Assessing the quality of reports of randomized clinical trials: is blinding necessary? Control Clin Trials 1996, 17:1-12.

13. R: A language and environment for statistical computing [http://www.r-project.org/]

14. Fletcher J: What is heterogeneity and is it important? BMJ 2007, 334:94-96

15. Higgins JP, Thompson SG: Quantifying heterogeneity in a meta-analysis Stat Med 2002, 21:1539-1558

16. Higgins JP, Thompson SG, Deeks JJ, Altman DG: Measuring inconsistency in meta-analyses. BMJ 2003, 327:557-560.

17. Deeks JJ, Higgins JPT, Altman DG: 9.5.2 Identifying and measuring heterogeneity. In Cochrane Handbook for Systematic Reviews of Interventions. Version 5-1-0. Edited by Higgins JPT, Green S. London: The Cochrane Collaboration; 2011. [http://www.cochrane-handbook.org]

18. Galbraith RF: A note on graphical presentation of estimated odds ratios from several clinical trials. Stat Med 1988, 7:889-894.

19. Deville WL, Buntinx F, Bouter LM, Montori VM, de Vet HC, van der Windt DA, Bezemer PD: Conducting systematic reviews of diagnostic studies: didactic guidelines. BMC Med Res Methodo/ 2002, 2:9.

20. Bender JS, Smith-Meek MA, Jones CE: Routine pulmonary artery catheterization does not reduce morbidity and mortality of elective vascular surgery: results of a prospective, randomized trial. Ann Surg 1997 226:229-236; discussion 236-237.

21. Benes J, Chytra I, Altmann P, Hluchy M, Kasal E, Svitak R, Pradl R, Stepan M: Intraoperative fluid optimization using stroke volume variation in high risk surgical patients: results of prospective randomized study. Crit Care 2010, 14:R118.

22. Bishop MH, Shoemaker WC, Appel PL, Meade P, Ordog GJ, Wasserberger J, Wo CJ, Rimle DA, Kram HB, Umali R, et al.: Prospective, randomized trial of survivor values of cardiac index, oxygen delivery, and oxygen consumption as resuscitation endpoints in severe trauma. J Trauma 1995, 38:780-787.

23. Bonazzi M, Gentile F, Biasi GM, Migliavacca S, Esposti D, Cipolla M, Marsicano M, Prampolini F, Ornaghi M, Sternjakob S, Tshomba Y: Impact of perioperative haemodynamic monitoring on cardiac morbidity after major vascular surgery in low risk patients. A randomised pilot trial. Eur J Vasc Endovasc Surg 2002, 23:445-451.

24. Cecconi M, Fasano N, Langiano N, Divella M, Costa MG, Rhodes A, Della Rocca G: Goal-directed haemodynamic therapy during elective total hip arthroplasty under regional anaesthesia. Crit Care 2011, 15:R132.

25. Challand C, Struthers R, Sneyd JR, Erasmus PD, Mellor N, Hosie KB, Minto G: Randomized controlled trial of intraoperative goal-directed fluid therapy in aerobically fit and unfit patients having major colorectal surgery. $\mathrm{Br} J$ Anaesth 2012, 108:53-62.

26. Chytra I, Pradl R, Bosman R, Pelnar P, Kasal E, Zidkova A: Esophageal Dopplerguided fluid management decreases blood lactate levels in multipletrauma patients: a randomized controlled trial. Crit Care 2007, 11:R24.

27. Donati A, Loggi S, Preiser JC, Orsetti G, Munch C, Gabbanelli V, Pelaia P, Pietropaoli P: Goal-directed intraoperative therapy reduces morbidity and length of hospital stay in high-risk surgical patients. Chest 2007, 132:1817-1824.

28. Forget $\mathrm{P}$, Lois F, de Kock M: Goal-directed fluid management based on the pulse oximeter-derived pleth variability index reduces lactate levels and improves fluid management. Anesth Analg 2010, 111:910-914.

29. Gan TJ, Soppitt A, Maroof M, el-Moalem H, Robertson KM, Moretti E, Dwane P, Glass PS: Goal-directed intraoperative fluid administration reduces length of hospital stay after major surgery. Anesthesiology 2002, 97:820-826

30. Harten J, Crozier JE, McCreath B, Hay A, McMillan DC, McArdle CS, Kinsella J: Effect of intraoperative fluid optimisation on renal function in patients undergoing emergency abdominal surgery: a randomised controlled pilot study (ISRCTN 11799696). Int J Surg 2008, 6:197-204.

31. Jhanji S, Vivian-Smith A, Lucena-Amaro S, Watson D, Hinds CJ, Pearse RM: Haemodynamic optimisation improves tissue microvascular flow and oxygenation after major surgery: a randomised controlled trial. Crit Care 2010, 14:R151.

32. Kapoor PM, Kakani M, Chowdhury U, Choudhury M, Lakshmy, Kiran U: Early goal-directed therapy in moderate to high-risk cardiac surgery patients. Ann Card Anaesth 2008, 11:27-34.

33. Lobo SM, Salgado PF, Castillo VG, Borim AA, Polachini CA, Palchetti JC, Brienzi SL, de Oliveira GG: Effects of maximizing oxygen delivery on morbidity and mortality in high-risk surgical patients. Crit Care Med 2000, 28:3396-3404.

34 Lopes MR, Oliveira MA, Pereira VO, Lemos IP. Auler JO, Jr Michard F: Goaldirected fluid management based on pulse pressure variation monitoring during high-risk surgery: a pilot randomized controlled trial. Crit Care 2007, 11:R100.

35. Mayer J, Boldt J, Mengistu AM, Rohm KD, Suttner S: Goal-directed intraoperative therapy based on autocalibrated arterial pressure waveform analysis reduces hospital stay in high-risk surgical patients: a randomized, controlled trial. Crit Care 2010, 14:R18.

36. McKendry M, McGloin H, Saberi D, Caudwell L, Brady AR, Singer M: Randomised controlled trial assessing the impact of a nurse delivered, flow monitored protocol for optimisation of circulatory status after cardiac surgery. BMJ 2004, 329:258-261.

37. Noblett SE, Snowden CP, Shenton BK, Horgan AF: Randomized clinical trial assessing the effect of Doppler-optimized fluid management on outcome after elective colorectal resection. Br J Surg 2006, 93:1069-1076.

38. Pearse R, Dawson D, Fawcett J, Rhodes A, Grounds RM, Bennett ED: Early goal-directed therapy after major surgery reduces complications and duration of hospital stay. A randomised, controlled trial [ISRCTN38797445]. Crit Care 2005, 9:R687-R693.

39. Pölönen P, Ruokonen E, Hippelainen M, Poyhonen M, Takala J: A prospective randomized study of goal-oriented hemodynamic therapy in cardiac surgical patients. Anesth Analg 2000, 90:1052-1059.

40. Valentine RJ, Duke ML, Inman MH, Grayburn PA, Hagino RT, Kakish HB, Clagett GP: Effectiveness of pulmonary artery catheters in aortic surgery: a randomized trial. J Vasc Surg 1998, 27:203-211; discussion 211-212.

41. Venn R, Steele A, Richardson P, Poloniecki J, Grounds M, Newman P: Randomized controlled trial to investigate influence of the fluid challenge on duration of hospital stay and perioperative morbidity in patients with hip fractures. Br J Anaesth 2002, 88:65-71.

42. Wakeling HG, McFall MR, Jenkins CS, Woods WG, Miles WF, Barclay GR, 
Fleming SC: Intraoperative oesophageal Doppler guided fluid management shortens postoperative hospital stay after major bowel surgery. Br J Anaesth 2005, 95:634-642

43. Wilson J, Woods I, Fawcett J, Whall R, Dibb W, Morris C, McManus E: Reducing the risk of major elective surgery: randomised controlled trial of preoperative optimisation of oxygen delivery. BMJ 1999, 318:1099-1103.

44. Futier E, Constantin JM, Petit A, Chanques G, Kwiatkowski F, Flamein R, Slim K, Sapin V, Jaber S, Bazin JE: Conservative vs restrictive individualized goaldirected fluid replacement strategy in major abdominal surgery: a prospective randomized trial. Arch Surg 2010, 145:1193-1200.

45. Jammer I, Ulvik A, Erichsen C, Lodemel O, Ostgaard G: Does central venous oxygen saturation-directed fluid therapy affect postoperative morbidity after colorectal surgery? A randomized assessor-blinded controlled trial. Anesthesiology 2010, 113:1072-1080

46. Lobo SM, Ronchi LS, Oliveira NE, Brandao PG, Froes A, Cunrath GS, Nishiyama KG, Netinho JG, Lobo FR: Restrictive strategy of intraoperative fluid maintenance during optimization of oxygen delivery decreases major complications after high-risk surgery. Crit Care 2011, 15:R226.

47. Schrier RW, Wang W: Acute renal failure and sepsis. N Eng/J Med 2004, 351:159-169.

48. Lipcsey M, Bellomo R: Septic acute kidney injury: hemodynamic syndrome, inflammatory disorder, or both? Crit Care 2011, 15:1008.

49. Langenberg C, Bellomo R, May C, Wan L, Egi M, Morgera S: Renal blood flow in sepsis. Crit Care 2005, 9:R363-R374.

50. Saotome T, Ishikawa K, May CN, Birchall IE, Bellomo R: The impact of experimental hypoperfusion on subsequent kidney function. Intensive Care Med 2010, 36:533-540.

51. Chua HR, Glassford N, Bellomo R: Acute kidney injury after cardiac arrest. Resuscitation 2012, 83:721-727.

52. Wan L, Bellomo R, May CN: A comparison of $4 \%$ succinylated gelatin solution versus normal saline in stable normovolaemic sheep: global haemodynamic, regional blood flow and oxygen delivery effects. Anaesth Intensive Care 2007, 35:924-931.

53. Legrand M, Mik EG, Balestra GM, Lutter R, Pirracchio R, Payen D, Ince C: Fluid resuscitation does not improve renal oxygenation during hemorrhagic shock in rats. Anesthesiology 2010, 112:119-127.

54. Dyson A, Bezemer R, Legrand M, Balestra G, Singer M, Ince C: Microvascular and interstitial oxygen tension in the renal cortex and medulla studied in a 4-h rat model of LPS-induced endotoxemia. Shock 2011, 36:83-89.

55. Rasmussen HH, Ibels LS: Acute renal failure. Multivariate analysis of causes and risk factors. Am J Med 1982, 73:211-218.

56. Liu YL, Prowle J, Licari E, Uchino S, Bellomo R: Changes in blood pressure before the development of nosocomial acute kidney injury. Nephrol Dial Transplant 2009, 24:504-511.

57. Badin J, Boulain T, Ehrmann S, Skarzynski M, Bretagnol A, Buret J, BenzekriLefevre D, Mercier E, Runge I, Garot D, Mathonnet A, Dequin PF, Perrotin D: Relation between mean arterial pressure and renal function in the early phase of shock: a prospective, explorative cohort study. Crit Care 2011, 15:R135.

58. Myles PS, Bellomo R: A pivotal trial of fluid therapy for major abdominal surgery: need and equipoise. Crit Care Resusc 2011, 13:278-280.

59. Herrler T, Tischer A, Meyer A, Feiler S, Guba M, Nowak S, Rentsch M, Bartenstein P, Hacker M, Jauch KW: The intrinsic renal compartment syndrome: new perspectives in kidney transplantation. Transplantation 2010, 89:40-46
60. Liu KD, Thompson BT, Ancukiewicz M, Steingrub JS, Douglas IS, Matthay MA Wright P, Peterson MW, Rock P, Hyzy RC, Anzueto A, Truwit JD: Acute kidney injury in patients with acute lung injury: impact of fluid accumulation on classification of acute kidney injury and associated outcomes. Crit Care Med 2011, 39:2665-2671.

61. Pearse RM, Belsey JD, Cole JN, Bennett ED: Effect of dopexamine infusion on mortality following major surgery: individual patient data metaregression analysis of published clinical trials. Crit Care Med 2008, 36:1323-1329.

62. Gattinoni L, Brazzi L, Pelosi P, Latini R, Tognoni G, Pesenti A, Fumagalli R: A trial of goal-oriented hemodynamic therapy in critically ill patients. $\mathrm{SvO}_{2}$ Collaborative Group. N Engl J Med 1995, 333:1025-1032.

63. Hayes MA, Timmins AC, Yau EH, Palazzo M, Hinds CJ, Watson D: Elevation of systemic oxygen delivery in the treatment of critically ill patients. NEng/J Med 1994, 330:1717-1722

64. Rivers E, Nguyen B, Havstad S, Ressler J, Muzzin A, Knoblich B, Peterson E, Tomlanovich M; Early Goal-Directed Therapy Collaborative Group: Early goaldirected therapy in the treatment of severe sepsis and septic shock. NEngl J Med 2001, 345:1368-1377.

65. Lin SM, Huang CD, Lin HC, Liu CY, Wang CH, Kuo HP: A modified goaldirected protocol improves clinical outcomes in intensive care unit patients with septic shock: a randomized controlled trial. Shock 2006 26:551-557.

66. de Oliveira CF, de Oliveira DS, Gottschald AF, Moura JD, Costa GA, Ventura AC Fernandes JC, Vaz FA, Carcillo JA, Rivers EP, Troster EJ: ACCM/PALS haemodynamic support guidelines for paediatric septic shock: an outcomes comparison with and without monitoring central venous oxygen saturation. Intensive Care Med 2008, 34:1065-1075.

67. Maitland K, Kiguli S, Opoka RO, Engoru C, Olupot-Olupot P, Akech SO, Nyeko R, Mtove G, Reyburn H, Lang T, Brent B, Evans JA, Tibenderana JK, Crawley J, Russell EC, Levin M, Babiker AG, Gibb DM: Mortality after fluid bolus in African children with severe infection. N Eng/ J Med 2011, 364:2483-2495.

68. Scandinavian Starch for Severe Sepsis/Septic Shock Trial (6S) NCT00962156 [http://clinicaltrials.gov/ct2/show/NCT00962156]

69. Crystalloid Versus Hydroxyethyl Starch Trials (CHEST) - NCT00935168 [http://clinicaltrials.gov/ct2/show/NCT00935168]

70. Protocolized Care for Early Septic Shock (ProCESS) - NCT00510835 [http://clinicaltrials.gov/ct2/show/NCT00510835]

71. Australasian Resuscitation in Sepsis Evaluation Randomised Controlled Trial (ARISE) - NCT00975793 [http://clinicaltrials.gov/ct2/show/NCT00975793]

72. A multicentre, randomised controlled trial of the clinical and costeffectiveness of early, goal-directed, protocolised resuscitation for emerging septic shock (ProMISe) - ISRCTN36307479

[http://www.controlled-trials.com/ISRCTN36307479]

73. Optimisation of peri-operative cardiovascular management to improve surgical outcome (OPTIMISE) - ISRCTN04386758 [http://www.controlled-trials.com/ISRCTN04386758/pearse]

doi:10.1186/cc11345

Cite this article as: Prowle JR, et al: Clinical review: Volume of fluid resuscitation and the incidence of acute kidney injury - a systematic review. Critical Care 2012, 16:230 